\title{
A three-phase trans-ethnic study reveals $B 7-H 3$ expression is a significant and independent biomarker associated with colon cancer overall survival
}

\author{
Yuan Gao ${ }^{1 \#}, \mathrm{Yu} \mathrm{Xu}^{2,3 \#}$, Meiqin Gao ${ }^{2,3}$, Aimin Huang ${ }^{2,3}$, Pan $\mathrm{Chi}^{1}$ \\ ${ }^{1}$ Department of Colorectal Surgery, Fujian Medical University Union Hospital, Fuzhou, China; ${ }^{2}$ Department of Pathology, School of Basic Medical \\ Sciences, Fujian Medical University, Fuzhou, China; ${ }^{3}$ Institute of Oncology of Fujian Medical University, Fuzhou, China \\ Contributions: (I) Conception and design: P Chi, Y Gao, Y Xu; (II) Administrative support: P Chi; (III) Provision of study materials or patients: P \\ Chi, A Huang; (IV) Collection and assembly of data: Y Gao, Y Xu; (V) Data analysis and interpretation: Y Gao, Y Xu; (VI) Manuscript writing: All \\ authors; (VII) Final approval of manuscript: All authors. \\ \#These authors contributed equally to this work. \\ Correspondence to: Pan Chi. Department of Colorectal Surgery, Fujian Medical University Union Hospital, 29 Xinquan Road, Fuzhou 350001 , China. \\ Email: chipan363@163.com.
}

Background: There have been inconsistent results and conflicting conclusions among the existing prognostic studies of $B 7-H 3$ expression in colon cancer patients. Therefore, the association between $B$ 7- $H 3$ expression and colon cancer survival has remained largely unclear.

Methods: We performed a three-phase and trans-ethnic prognostic study of $B 7-H 3$ expression in colon cancer patients involving perhaps the largest population to date. In the discovery phase, we utilized a Cox proportional hazards model adjusted for covariates to test the association between $B 7-H 3$ expression and colon cancer overall survival (OS) time in a European population from The Cancer Genome Atlas (TCGA) cohort $(n=433)$. In the validation phase I, the association was replicated in a European population from Gene Expression Omnibus (GEO) cohort ( $\mathrm{n}=811)$. In the validation phase II, we again confirmed the significant association in an Asian population from Fujian Medical University Union Hospital (UNION) cohort ( $\mathrm{n}=179)$. Furthermore, a series of Kaplan-Meier analysis, bioinformatics analysis of tumor immune microenvironment (TIME), and immune checkpoint prognostic prediction analysis, as well as sensitivity analysis, were also conducted.

Results: Highly expressed $B 7-H 3$ was a significant and robust biomarker to colon cancer survival, with a large hazard ratio (HR) $\left[\mathrm{HR}_{\mathrm{TCGA}}=4.60,95 \%\right.$ confidence interval $(\mathrm{CI}): 2.15$ to $9.83, \mathrm{P}=8.37 \times 10^{-05} ; \mathrm{HR}_{\mathrm{GEO}}$ $=1.47,95 \%$ CI: 1.12 to $1.94, \mathrm{P}=0.0056 ; \mathrm{HR}_{\mathrm{UNION}}=1.63,95 \% \mathrm{CI}: 1.36$ to $1.95, \mathrm{P}=7.91 \times 10^{-08}$ ]. We detected an involvement of B7-H3 in the tumor immune microenvironment (TIME). Meanwhile, B7-H3 was significantly and weakly correlated with 6 out of 27 well-recognized immune checkpoint genes. Even after adjusting for effects of other immune checkpoint genes, $\mathrm{B} 7-\mathrm{H} 3$ still exhibited a harmful effect on colon cancer survival using samples from TCGA and GEO cohorts (HR =1.47, 95\% CI: 1.07 to 2.02, P=0.0184), indicating that it was an independent prognostic factor of colon cancer. We also proposed an immune checkpoint prognostic risk score which possessed the capability to identify colon cancers with high risk of mortality.

Conclusions: The expression of $B 7-H 3$ is a significant, robust, and independent prognostic factor to colon cancer OS.

Keywords: $B 7-H 3$; gene expression; colon cancer; overall survival; prognostic factor

Submitted Nov 05, 2021. Accepted for publication Dec 20, 2021.

doi: 10.21037/jgo-21-821

View this article at: https://dx.doi.org/10.21037/jgo-21-821 


\section{Introduction}

Colon cancer is a common malignant tumor of the digestive system, which is the third and second most commonly diagnosed cancer in men and women worldwide, respectively, with 1.15 million new cases and 576,858 deaths in 2020 (1). Even though the therapeutic effect of colon cancer has greatly improved with the development of medical technology, the prognosis of colon cancer is still poor (2). Immunotherapy, which boosts the immune system works to find and attack cancer cells, envisions a novel approach to cancer therapy (3). The immune system consists of a complex set of molecular interactions that are regulated by immune checkpoints and are named differentiated clusters based on their order of discovery $(4,5)$. Immune checkpoint pathways are often activated to inhibit nascent anti-tumor immune responses to tumor cells, and immune checkpoint therapy enhances the immune activity against tumors by blocking or stimulating these pathways $(6,7)$, including the most widely studied and well-recognized pathways of cytotoxic $\mathrm{T}$ lymphocyte-associated molecule-4 (CTLA-4), programmed cell death receptor-1 (PD-1), and programmed cell death ligand 1 (PD-L1). Inhibitory immune checkpoints (e.g., PD-L1, PD-1, CTLA-4, and B7-H3) bind with their associated ligands to induce an inhibitory response and prevent the cascade of stimulatory immune checkpoint signals that activate $T$ cell activity $(8,9)$. Therefore, those immune checkpoints are consequentially associated with tumor prognosis and response to treatment.

Nevertheless, the association between B7-H3 (B7 Homolog 3) and colon cancer survival still remains unclear. Also known as $\mathrm{CD} 276, \mathrm{B7}-\mathrm{H} 3$ is a costimulatory molecule belonging to the B7-CD28 family. It is a membrane protein encoded on chromosome $15(10,11)$, and is widely expressed in heart, thymus, prostate, testis, uterus, placenta, spleen, liver, pancreas, small intestine, and colon in both normal and tumor tissues (12-14). An array of studies have indicated that highly expressed $B 7-H 3$ promotes tumor progression and metastasis, as well as being associated with poor prognosis in various of cancers, including glioma (15), hepatocellular carcinoma (16), lung cancer (17), breast cancer (18), osteosarcoma (19), cutaneous melanoma (20), and pancreatic cancer (21). However, the results of existing prognostic studies of $\mathrm{B} 7-\mathrm{H} 3$ expression and colon cancer (22-37) have been inconsistent and have presented conflicting conclusions; thus, we believe there is an urgent need to elaborate the effect of $\mathrm{B} 7-\mathrm{H} 3$ on colon cancer survival. Besides, various technical bottlenecks of previous studies need to be addressed: (I) there have been very few European studies $(22,23,29)$, and majority of these studies were conducted among Asian populations (24-28,30-37); (II) no study so far has focused on the non-linear effects of $B 7-H 3$ on colon cancer survival, with previous studies simply having tested its linear effect; (III) there has been no independent validation of $\mathrm{B} 7-\mathrm{H} 3$, the association has consistently been evaluated in a single population; and (IV) there have been no trans-ethnic population studies of $\mathrm{B} 7-\mathrm{H} 3$ thus far.

Hence, we performed a 3-phase designed and transethnic study to test and confirm the prognostic effect of $B 7-$ $H 3$ expression on colon cancer survival using 3 independent cohorts comprising European and Asian populations, followed by a series of Kaplan-Meier (K-M) analysis, bioinformatics analysis of tumor immune microenvironment (TIME), and immune checkpoint prognostic prediction analysis, along with sensitivity analysis. We present the following article in accordance with the REMARK reporting checklist (available at https://dx.doi.org/10.21037/ jgo-21-821) (38).

\section{Methods}

\section{Study populations}

We utilized European colon cancer patients from The Cancer Genome Atlas (TCGA) and Gene Expression Omnibus (GEO) cohorts, as well as Asian colon cancer patients recruited from Fujian Medical University Union Hospital (UNION) to evaluate the association between gene expression level of $B 7-H 3$ and overall survival (OS) of colon cancer (Table 1).

\section{TCGA}

Gene expression profiles (platform: Illumina HiSeq 2000 RNA Sequencing; San Diego, CA, USA) and clinical data of colon cancer patients were obtained from TCGA (https://portal.gdc.cancer.gov/) database in July 2021, including 471 tumor tissues and 41 adjacent-normal tissues. A total of 433 participants (95 deceased and 338 alive) with complete clinical and OS time were reserved for subsequent association analysis. The gene expression level was measured by fragments per kilobase of transcript per million fragments (FPKM) value and $\log 2$ transformed before analysis. Unqualified probes were excluded if they meet any of the quality control (QC) criteria: (I) high missing rates $(>30 \%)$; or (II) coefficient of variance (CV) 
Table 1 Demographic and clinical descriptions of colon cancer patients in TCGA, GEO, and UNION cohorts

\begin{tabular}{|c|c|c|c|}
\hline Variable & TCGA $(n=433)$ & GEO $(n=811)$ & UNION $(n=179)$ \\
\hline \multicolumn{4}{|l|}{ Age group, n (\%) } \\
\hline$<65$ years & $169(39.03)$ & $325(40.07)$ & $126(70.39)$ \\
\hline$\geq 65$ years & $264(60.97)$ & 485 (59.80) & $53(29.61)$ \\
\hline \multicolumn{4}{|l|}{ Gender, n (\%) } \\
\hline Female & $200(46.19)$ & $370(45.62)$ & 89 (49.72) \\
\hline Male & $233(53.81)$ & $441(54.38)$ & $90(50.28)$ \\
\hline Height $(\mathrm{cm})$, mean $\pm S D$ & $168.52 \pm 12.32$ & - & $163.09 \pm 7.82$ \\
\hline \multicolumn{4}{|l|}{ T stage, n (\%) } \\
\hline T0/Tis & $1(0.23)$ & $4(0.69)$ & $0(0.00)$ \\
\hline $\mathrm{T} 1$ & $11(2.54)$ & $12(2.07)$ & $0(0.00)$ \\
\hline $\mathrm{T} 2$ & 75 (17.32) & $48(8.29)$ & $0(0.00)$ \\
\hline T3 & $296(68.36)$ & $376(64.94)$ & 34 (18.99) \\
\hline $\mathrm{T} 4$ & $50(11.55)$ & $119(20.55)$ & $145(81.01)$ \\
\hline Unknown & 0 & 252 & 0 \\
\hline \multicolumn{4}{|l|}{$\mathrm{N}$ stage, $\mathrm{n}(\%)$} \\
\hline No & $254(58.66)$ & $311(53.71)$ & $32(17.88)$ \\
\hline Unknown & 0 & 252 & 0 \\
\hline \multicolumn{4}{|l|}{ M stage, n (\%) } \\
\hline Mo & $320(73.90)$ & $496(85.66)$ & $0(0.00)$ \\
\hline M1 & $61(14.09)$ & $61(10.54)$ & 179 (100.00) \\
\hline MX & $45(10.39)$ & $2(0.35)$ & $0(0.00)$ \\
\hline Unknown & 7 & 252 & 0 \\
\hline \multicolumn{4}{|l|}{ Clinical stage, n (\%) } \\
\hline I & $73(16.86)$ & $65(8.01)$ & $0(0.00)$ \\
\hline II & $165(38.11)$ & $341(42.05)$ & $0(0.00)$ \\
\hline III & $123(28.41)$ & $285(35.14)$ & $0(0.00)$ \\
\hline IV & 61 (14.09) & $116(14.30)$ & $179(100.00)$ \\
\hline Unknown & 11 & 4 & 0 \\
\hline
\end{tabular}

Table 1 (continued) 
Table 1 (continued)

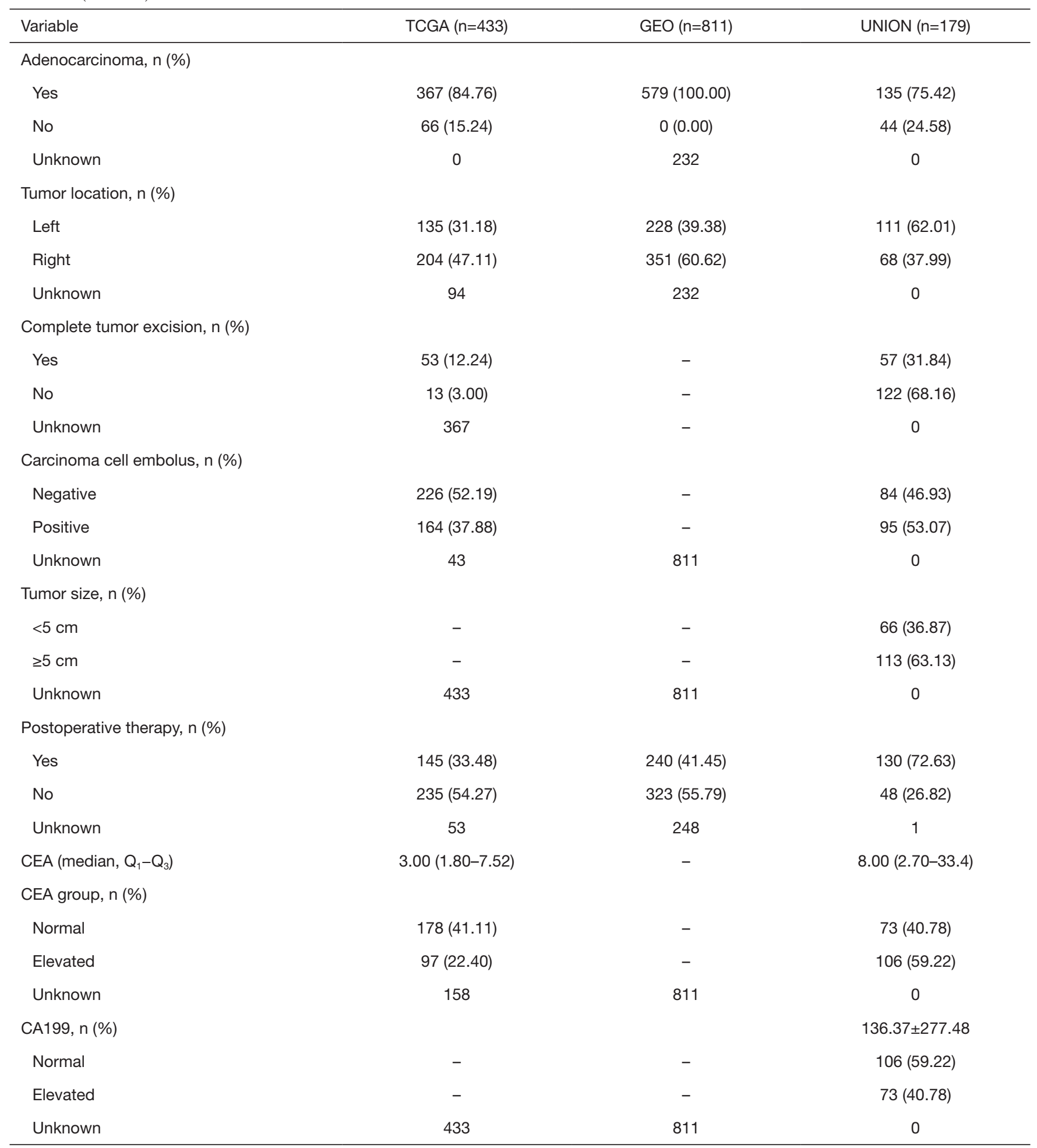

Table 1 (continued) 
Table 1 (continued)

\begin{tabular}{lccc}
\hline Variable & TCGA $(n=433)$ & GEO $(n=811)$ & UNION $(n=179)$ \\
\hline Median survival months & & & \\
Median $(95 \% \mathrm{Cl})$ & $93(66-\mathrm{NA})$ & $135(106-\mathrm{NA})$ & $16(11-20)$ \\
Deaths, $\mathrm{n}(\%)$ & $95(21.94)$ & $287(35.39)$ & $137(76.54)$ \\
\hline
\end{tabular}

NA, not available; T, tumor; N, node; M, metastasis; CEA, carcinoembryonic antigen; TCGA, The Cancer Genome Atlas; GEO, Gene Expression Omnibus; UNION, Fujian Medical University Union Hospital.

$<5 \%$ (Figure S1). The data used in this study comply with the requirements of TCGA official published data and are publicly available.

\section{GEO}

A total of 811 colon cancer patients with complete clinical and OS information, as well as gene expression data, were acquired from the GEO (https://www.ncbi.nlm.nih.gov/ geo/) database, including GSE39582, GSE17536, and GSE17537. Gene expression data was profiled by GPL570 Affymetrix Human Genome U133 Plus 2.0 Array platform (Affymetrix, Santa Clara, CA, USA). The raw intensity values were background corrected, $\log 2$ transformed, and then quantile normalized. Next, the expression values were derived by robust multi-array average (RMA) method, an algorithm exclusively designed to create an expression matrix from Affymetrix platform. Redundant probes were collapsed, and then annotated to human gene symbols prior to analysis. We applied the same QC criteria for the GEO data.

\section{UNION}

Colon cancer patients in the UNION cohort were recruited from January 2010 to January 2018. Patients were admitted for surgical treatment of colorectal cancer. Routine preoperative examinations were performed to exclude patients with organic lesions of the heart, lung, liver, and others. Additionally, patients (I) who did not receive surgery but only a simple ileostomy; (II) who had intestinal obstruction, intestinal perforation, intestinal bleeding, or required emergency surgical resection; (III) who had simultaneous polygenic carcinoma of the colon, heterogeneous polygenic carcinoma of the colon, and familial adenomatous polyposis; (IV) who presented other malignant tumors or died due to postoperative complications, were further excluded. Finally, 179 patients were included in the study. The expression level of $B 7-H_{3}$ in tumor cells was quantified by immunohistochemistry
(IHC). Briefly, Slides (4- $\mu$ m thick consecutive paraffin sections) from the blocks with the highest tumor content for each sample were used for IHC staining, immersed in xylene, rehydrated through graded concentrations of ethanol followed by phosphate-buffered saline (PBS) buffer, and deionized water for $5 \mathrm{~min}$ each. Slides were then heated to $100{ }^{\circ} \mathrm{C}$ for $20 \mathrm{~min}$ in a $\mathrm{pH} 9$ Tris-based solution. All slides were incubated with the primary antibodies for $60 \mathrm{~min}$ at $37^{\circ} \mathrm{C}$ for $1 \mathrm{~h}$ (dilutions: Human B7-H3 Antibody 1:300, R\&D Systems, Minneapolis, MN, USA) and were then washed. A secondary antibody followed by incubation with rabbit anti-goat IgG and an avidin-biotin complex (Boster, Wuhan, China) was added for $30 \mathrm{~min}$ and the slides were again washed. The sections were processed with the universal SP Elivision-plus kit (Maixin Bio, Fuzhou, China). Finally, the sections were counterstained with hematoxylin (39). The percentages of $B 7-H 3$ in 3 representative high-power fields of individual samples were analyzed for intensity of $B 7$ H3 membranous expression and were scored as $0(<5 \%$ of B7-H3), 1 (5-25\%), 2 (>25-50\%), or 3 (>50\%). Individual samples were evaluated by at least 2 pathologists in a blinded manner, and those samples with inconsistent scores were further discussed and decided. A score of 0 or 1 with $B 7-H 3$ on IHC was regarded as low $B 7-H 3$ expression group, and 2 or 3 staining as high $B 7-H 3$ expression group. This study was approved by the institutional review board of Fujian Medical University Union Hospital (No. 2018YF024-01). All participants or their surrogates provided their written informed consent. All procedures performed in this study involving human participants were in accordance with the Declaration of Helsinki (as revised in 2013).

\section{Statistical analysis}

\section{Three-phase study of B7-H3 expression and colon} cancer OS

The analysis workflow is presented in Figure 1. We adopted a 3-phase trans-ethnic study to evaluate the association 


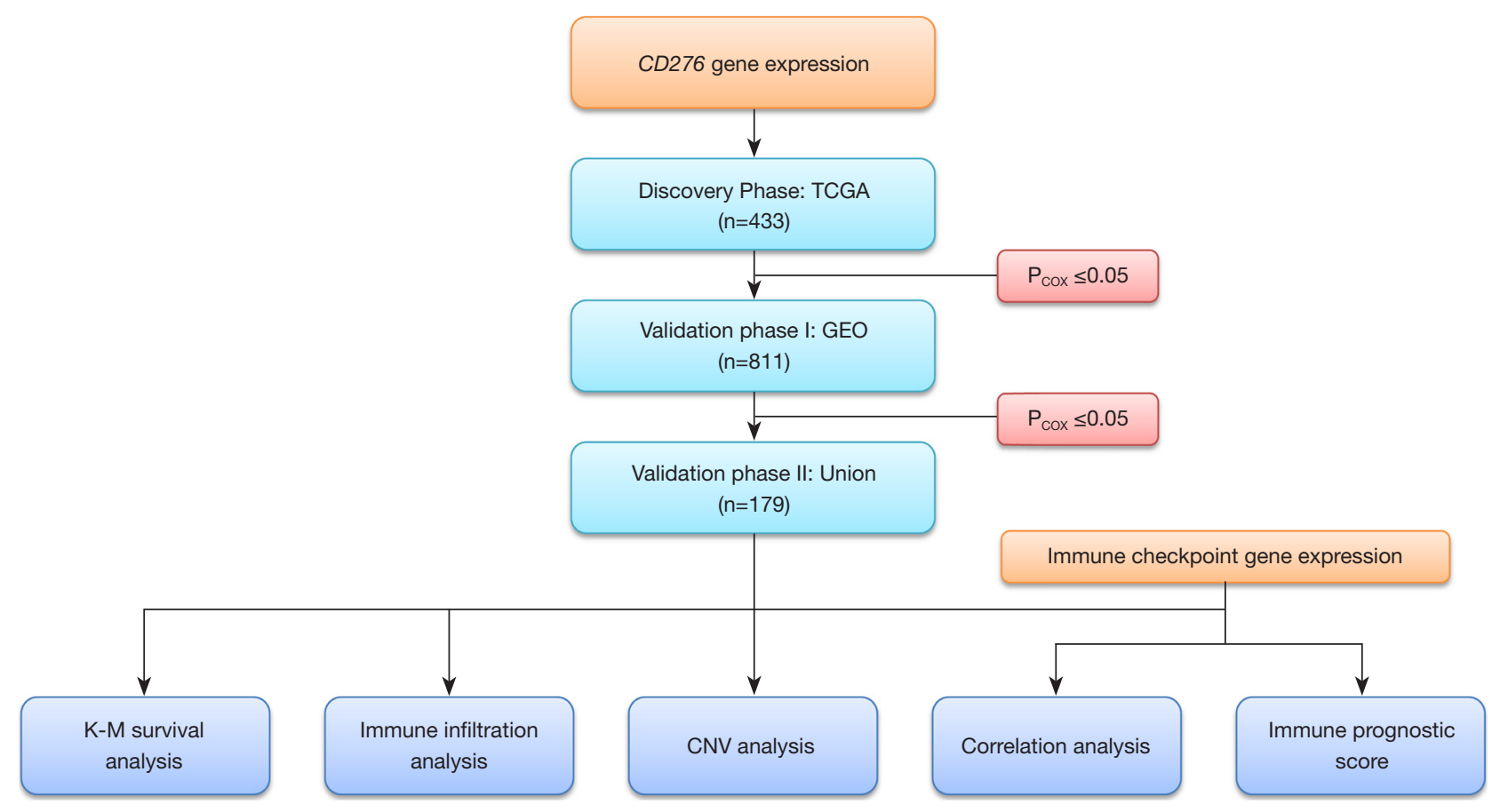

Figure 1 Flow chart of study design and statistical analysis.

between expression of $B 7-H 3$ and colon cancer OS. In the discovery phase, we used a restricted cubic spline (RCS) regression model by setting the median expression of $\mathrm{B} 7-\mathrm{H3}$ as a reference point to investigate the potential non-linear effect of $B 7-H 3$ on OS using a European population from the TCGA cohort. The RCS are a smoothly joined sum of polynomial functions that do not assume linearity of the relationship between variable and the response (40). This technique provides great flexibility for fitting data and modeling complex relationships between survival outcome and the variable of interest, while adjusting for other covariates. Furthermore, an RCS regression model can avoid arbitrary categorization of the continuous measures and permit identification of the risk function inflexion point. Then, the $B 7-H 3$ expression was stratified by knot information into different groups (41). Finally, the association between $\mathrm{B} 7-\mathrm{H} 3$ and survival was tested using a Cox proportional hazards model adjusted for covariates. These covariates were significant variables screened out by step forward regression model with $\mathrm{P}$ value of entry $\leq 0.05$ and $\mathrm{P}$ value of remove $>0.05$, among a series of demographic and clinical variables. As a result, age, gender, and clinical stage were adjusted in the Cox proportional hazards model (Tables S1,S2). Proportional hazard assumption was confirmed based on Schoen's method (42). The association strength was measured by hazard ratio (HR) and $95 \%$ confidence interval (CI).

In the validation phase I, we used the RCS regression model again to evaluate the non-linear effect of $\mathrm{B} 7-\mathrm{H} 3$ on colon cancer survival using a European population from the GEO cohort. The Cox proportional hazards model was utilized to test the association between $B 7-H 3$ and survival, adjusted for the same covariates aforementioned in the discovery phase.

In the validation phase II, we directly tested the grade level of $B 7-H 3$ using Cox proportional hazards regression model using an Asian population from the UNION cohort. Since all participants were in M1 stage, the model was adjusted for age, gender, tumor (T) stage, and node (N) stage. Finally, meta-analyses using both fixed-effects and random-effects models were applied in our study to combine results from 3 phases by $\mathrm{R}$ package meta (https:// cran.r-project.org/web/packages/meta/index.html).

\section{Supplementary analyses of B7-H3}

Furthermore, we performed a series of supplementary analyses. First, K-M survival curves were drawn to represent the survival difference between patients with 
different expression levels (43). The cutoff values of $B 7-H 3$ expression were defined based on using $\mathrm{R}$ package survMisc (https://cran.r-project.org/web/packages/survMisc/index. html), which used log-rank test to find the optimal cut-off value, in TCGA and GEO cohorts, respectively. According to these cutoff values, these participants were divided into a high and low expression group, their survival differences were further compared using Cox proportional hazards regression model adjusted for covariates.

Second, bioinformatics analysis of $B 7-H 3$ including immune infiltration analysis, somatic copy number alteration (SCNA) analysis, and correlation analysis were performed using TIMER2.0, which is a freely available web server to the research community (44). The TIMER2.0 resource uses RNA-seq expression profiles to detect and quantify the situation of immune cell infiltration in tumor tissues, so as to determine the relationship between tumor and immune cells in TCGA colon cancer database (45). (I) In immune infiltration analysis, the scatterplots were displayed, showing the association between $B 7$ $H 3$ expression and immune infiltration level in colon cancer tumors using Spearman correlation analysis. (II) In SCNA analysis, SCNAs were categorized into different groups, including deep deletion (-2), arm-level deletion $(-1)$, diploid/normal (0), arm-level gain $(+1)$, and high amplification $(+2)$. Box plots were presented to show the distributions of each immune subset (B cells, $\mathrm{CD} 8^{+} \mathrm{T}$ cells, $\mathrm{CD}^{+} \mathrm{T}$ cells, macrophages, neutrophils, and dendritic cells) at each copy number status in colon cancer. The infiltration level for each category was compared with the normal using a 2-sided Wilcoxon rank-sum test. (III) In correlation analysis, we further evaluated the association between $B 7$ $H 3$ and 27 well-recognized immune checkpoint genes (46).

Finally, we proposed an immune checkpoint prognostic score of colon cancer using overall participants from TCGA and GEO cohorts to distinguish patients at high risk of mortality. These well-recognized immune checkpoint genes associated with colon cancer survival were screened out by forward stepwise regression model adjusted for age, gender, clinical stage, study site and $B 7-H 3$, with $\mathrm{P}$ value of entry $\leq 0.05$ and $\mathrm{P}$ value of remove $>0.05$. Patients were then categorized into high and low risk groups by median value, and K-M survival curves between 2 groups were tested. Sensitivity analyses were also performed stratified by age, gender, and clinical stage. The time-dependent receiver operating characteristic (ROC) curve and area under the ROC curve (AUC) were used to measure the prediction ability (47). Statistical analyses were performed using R 268 version 3.4.4 (The R Foundation of Statistical Computing, 269 Vienna, Austria).

\section{Results}

After quality control, 3 datasets were composed of with 433 participants in TCGA cohort, 811 participants in the GEO cohort, and 179 participants in the UNION cohort for further association analysis. Demographic and clinical characteristics of these participants were described as mean \pm SD for continuous variables and number (\%) for categorical variables in Table 1 .

\section{Clinical experimental studies of B7-H3 and colon cancer}

First, the expression level of $\mathrm{B} 7-\mathrm{H} 3$ in colon cancer and adjacent normal tissues were evaluated by differential analysis of gene expression data in TCGA and GEO cohort. Compared with the adjacent normal tissue, tumor tissue had high-expressed level of $B 7-H 3\left(\mathrm{P}=1.18 \times 10^{-21}\right)$. Besides, there was significant association between $B 7-H 3$ expression and clinicopathological factors (age: $\mathrm{P}=0.029$; clinical stage: $\mathrm{P}=0.002$ ), which were shown in boxplot (Figure S2).

\section{B7-H3 was significantly and robustly associated colon cancer $\mathrm{OS}$}

In the discovery phase, we observed the apparent non-linear association pattern between $B 7-H 3$ and OS time of colon cancer patients from cubic spline regression model using European population in TCGA (Figure 2). This suggested that the inflection points were $3.7,4.1$, and 4.6. The covariates-adjusted Cox proportional hazards regression model indicated $\mathrm{B} 7-\mathrm{H} 3$ was a significant risk factor of colon cancer survival $\left(\mathrm{HR}_{\mathrm{TCGA}}=4.60,95 \% \mathrm{CI}: 2.15\right.$ to $\left.9.83, \mathrm{P}=8.37 \times 10^{-05}\right)$. In the validation phase $\mathrm{I}$, there was an approximate linear association pattern between $B 7-H 3$ and colon cancer survival derived from cubic spline regression model using European population in GEO. By Cox model, $B 7-H 3$ was still significantly correlated with a worse prognosis $\left(\mathrm{HR}_{\mathrm{GEO}}=1.47,95 \% \mathrm{CI}: 1.12\right.$ to $\left.1.94, \mathrm{P}=0.0056\right)$. In validation phase II, we again confirmed that $B 7-H 3$ was a significant prognostic factor of colon cancer survival using an Asian population in UNION $\left(\mathrm{HR}_{\mathrm{UNION}}=1.63,95 \%\right.$ CI: 1.36 to $\left.1.95, \mathrm{P}=7.91 \times 10^{-08}\right)$. Finally, meta-analysis of 3 phases ensured the significant association regardless of 

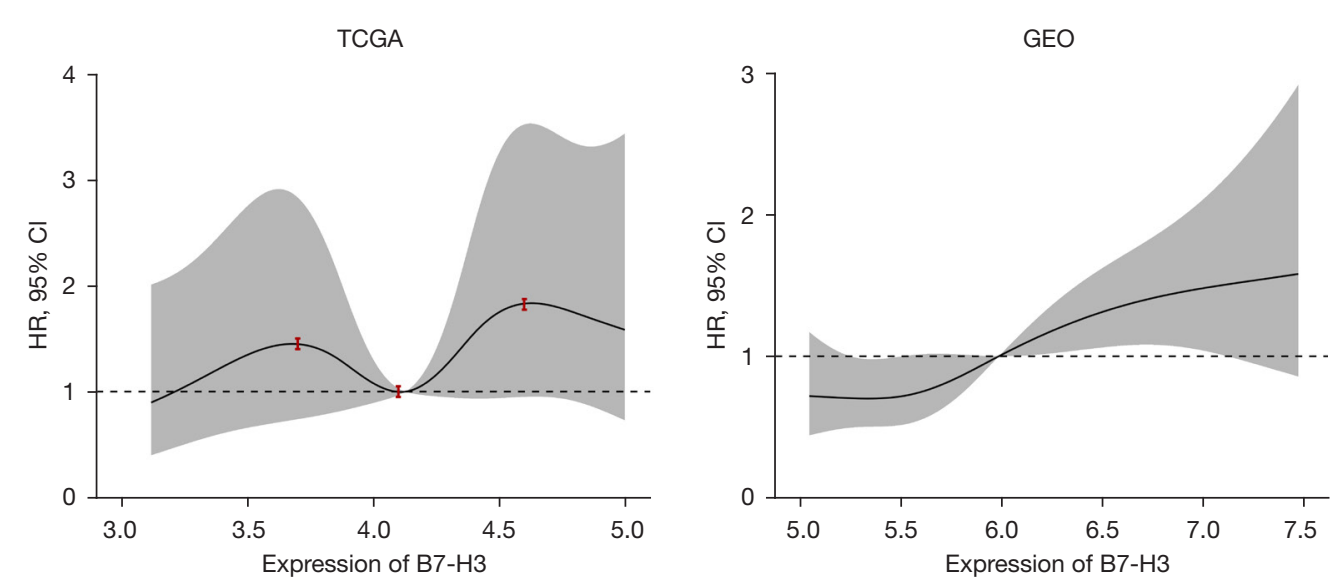

Figure 2 The apparent non-linear and approximate linear association pattern between $B 7-H 3$ and OS time of colon cancer patients derived from cubic spline regression model. The inflection points of expression of B7-H3 were 3.7, 4.1, and 4.6 in TCGA cohort. OS, overall survival; TCGA, The Cancer Genome Atlas.

Table 2 Association results of $B 7-H 3$ expression and overall survival time of colon cancer patients derived from Cox proportional hazards model in TCGA, GEO, and UNION cohorts

\begin{tabular}{|c|c|c|c|c|c|c|}
\hline Variable & \multicolumn{3}{|c|}{ Unadjusted model } & \multicolumn{3}{|c|}{ Adjusted model } \\
\hline TCGA & 3.240 & $1.514-6.933$ & 0.0024 & 4.598 & $2.150-9.833$ & 8.37E-05 \\
\hline GEO & 1.332 & $1.016-1.746$ & 0.0380 & 1.472 & $1.119-1.937$ & 0.0056 \\
\hline UNION & 1.553 & $1.317-1.831$ & $1.7 \mathrm{E}-07$ & 1.631 & $1.364-1.950$ & 7.91E-08 \\
\hline Fixed-effects model & 1.529 & $1.331-1.756$ & $1.91 \mathrm{E}-09$ & 1.646 & $1.421-1.907$ & $2.85 \mathrm{E}-11$ \\
\hline Random-effects model & 1.585 & $1.203-2.087$ & 0.0011 & 1.846 & $1.281-2.661$ & 0.0010 \\
\hline Test of heterogeneity & & & 0.0919 & & & 0.0217 \\
\hline
\end{tabular}
a fixed-effects model $\left(\mathrm{HR}_{\text {fixed }}=1.65,95 \% \mathrm{CI}: 1.42\right.$ to 1.91 ,
$\left.\mathrm{P}=2.85 \times 10^{-11}\right)$ or random-effects model $\left(\mathrm{HR}_{\text {random }}=1.85\right.$,
$95 \%$ CI: 1.28 to $2.66, \mathrm{P}=0.0010)($ Table 2$)$.

\section{K-M survival analysis}

The optimal cutoff value of $B 7-H 3$ which corresponded to the highest log-rank test score was 4.317 and 5.864 in TCGA and GEO, respectively. Therefore, participants in TCGA and GEO were divided into high and low expression groups. In UNION, participants with the grade levels ( 0 and 1$)$ of $B 7-H 3$ expression were categorized into low expression group and the others were in high expression group. The survival curves were significantly 328 separated between the 2 groups across 3 datasets (Figure 3), 329 indicating that high expression of $\mathrm{B} 7-\mathrm{H} 3$ had harm effect 330 on colon cancer survival $\left(\mathrm{HR}_{\mathrm{TCGA}}=1.63,95 \% \mathrm{CI}: 1.07\right.$ to 331 2.49, $\mathrm{P}=2.22 \times 10^{-02} ; \mathrm{HR}_{\mathrm{GEO}}=1.75,95 \% \mathrm{CI}: 1.26$ to $2.42,332$ $\mathrm{P}=7.62 \times 10^{-04} ; \mathrm{HR}_{\mathrm{UNION}}=2.36,95 \% \mathrm{CI}: 1.64$ to $3.40,333$ $\left.\mathrm{P}=4.21 \times 10^{-06}\right)$. 334

\section{Bioinformatics analysis}

Cancer cells within the TIME and neighboring tumorassociated noncancerous cells play an important role in tumor biology. The immune infiltration analysis indicated 

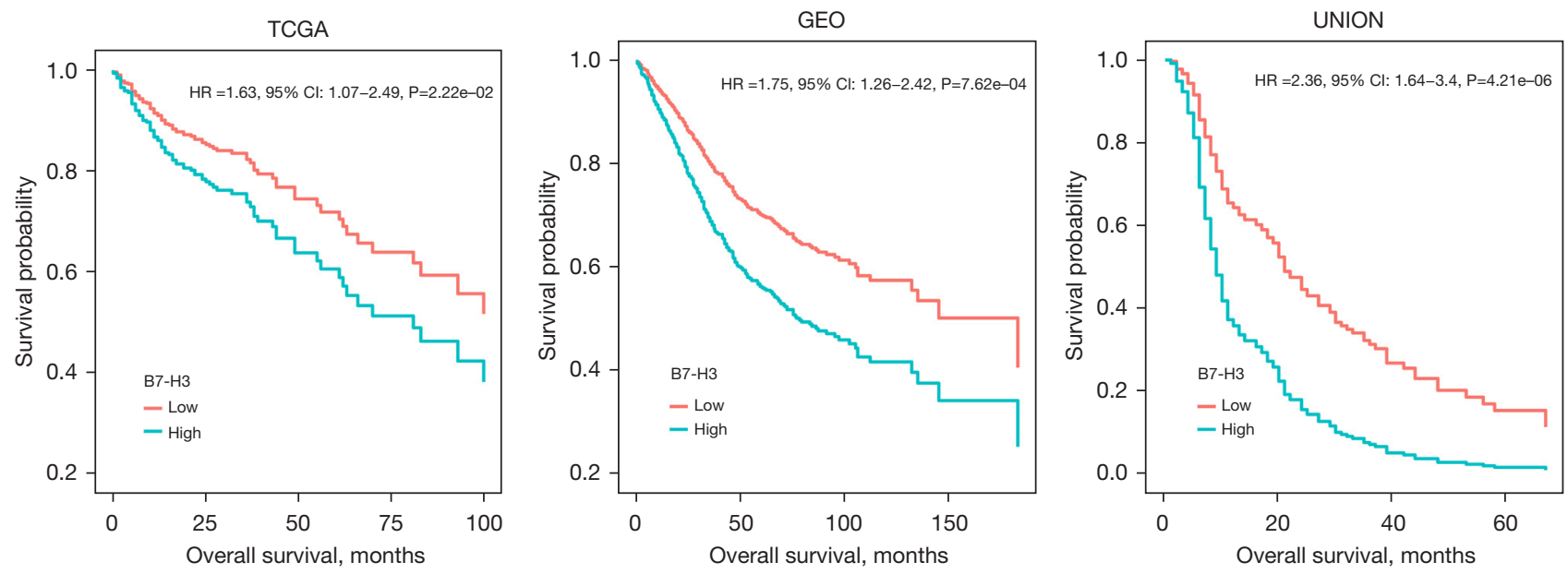

Figure $3 \mathrm{~K}-\mathrm{M}$ survival curves of colon cancer patients with high and low gene expression level of B7-H3 in TCGA, GEO, and UNION cohorts. K-M, Kaplan-Meier; TCGA, The Cancer Genome Atlas; GEO, Gene Expression Omnibus; UNION, Fujian Medical University Union Hospital.

that $B 7-H 3$ expression had a significant negative correlation with immune infiltration level for tumor purity and B cells, but a significant positive correlation with that for $\mathrm{CD}^{+}$ $\mathrm{T}$ cells, macrophages, neutrophils, and dendritic cells (Figure 4). Besides, the infiltration levels were significantly distributed among different groups of SCNAs of $B 7-H_{3}$ (Figure 5), including $\mathrm{B}$ cell $(\mathrm{P}<0.05), \mathrm{CD}^{+} \mathrm{T}$ cell $(\mathrm{P}<0.001)$, neutrophil $(\mathrm{P}<0.01)$, and dendritic cell $(\mathrm{P}<0.001)$. In addition, $B 7-H 3$ was significantly and weakly correlated with 6 out of 27 well-recognized immune checkpoint genes (Figure S3), including PVRL2 $\left(\rho=0.47, \mathrm{P}=1.90 \times 10^{-05}\right), P V R$ $\left(\rho=0.43, P=8.31 \times 10^{-5}\right), \operatorname{IGSF11}\left(\rho=0.29, \mathrm{P}=9.74 \times 10^{-03}\right)$, CEACAM1 $\left(\rho=0.35, \mathrm{P}=1.90 \times 10^{-03}\right)$, PTDSS1 $(\rho=0.43$, $\left.\mathrm{P}=1.04 \times 10^{-04}\right)$, and HMGB1 $\left(\rho=0.49, \mathrm{P}=6.61 \times 10^{-05}\right)$.

\section{Immune checkpoint prognostic score of colon cancer}

We performed stepwise regression model to screen other immune checkpoint genes using Cox model adjusted for covariates and $\mathrm{B} 7-\mathrm{H} 3$ expression and identified 7 more genes associated with colon cancer survival (Table S3). In this multivariable Cox model, $B 7-H 3$ was still significant ( $\mathrm{HR}=1.47,95 \% \mathrm{CI}: 1.07$ to $2.02, \mathrm{P}=0.0184$ ), indicating it was an independent risk factor of colon cancer prognosis. Then, we proposed an immune checkpoint prognostic score of colon cancer, which was a weighted linear combination of 8 significant genes. The weight of each gene was defined as the corresponding estimated $\log$ hazard ratio $[\ln (\mathrm{HR})]$ derived from the multivariable Cox model. The immune checkpoint prognostic score could distinguish patients at high risk of mortality in all subpopulations stratified by age (Figure S4), gender (Figure S5), and clinical stages (Figure S6). These 8 immune checkpoint genes slightly improved the prognostic prediction ability for 3-year survival (2.38\%) and 5-year survival (1.63\%), respectively. Hence, the prognostic model of colon cancer achieved an acceptable accuracy $\left(\mathrm{AUC}_{3 \text {-year }}=0.74\right.$ and $\left.\mathrm{AUC}_{3 \text {-year }}=0.72\right)$ (Figure S7).

\section{Discussion}

In recent years, previous studies of $\mathrm{B} 7-\mathrm{H} 3$ have mainly focused on the its expression and role in different types of cancers (48). Elevated expression of $B 7 H 3$ has been found in serum of patients with malignant tumors, which is related to clinical stage and progression of cancer $(49,50)$, since $B 7-H 3$ appears to be correlated with different proteins that contribute to tumor migration, invasion and angiogenesis $(51,52)$. Besides, $B 7-H 3$ blocking in combination with chemotherapy is a promising treatment option based on preclinical animal studies (53).

Currently, some previous studies have described a significant correlation between tumor expression of $\mathrm{B} 7-\mathrm{H} 3$ and prognosis $(14,54)$, whereas, majority of them reported that high tumor $B 7-H 3$ expression was associated with more advanced disease (31), increased risk of recurrence (35) or 


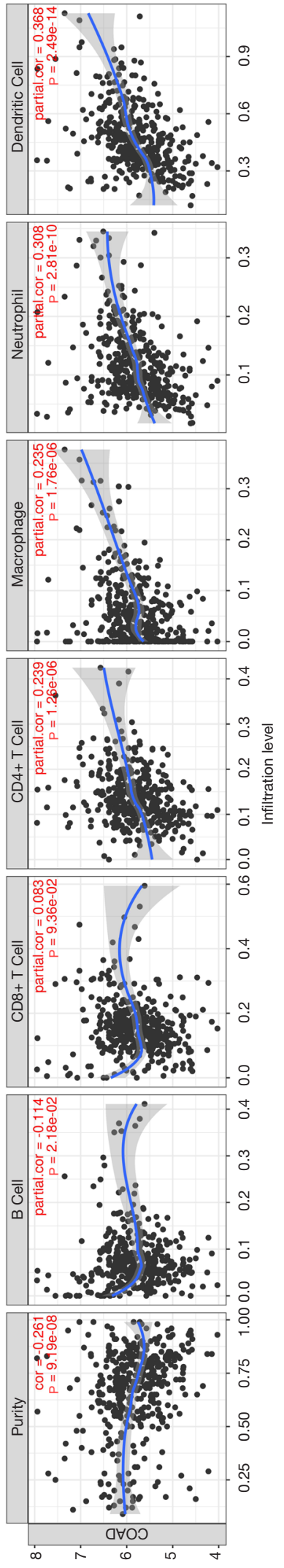

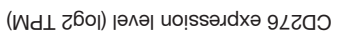

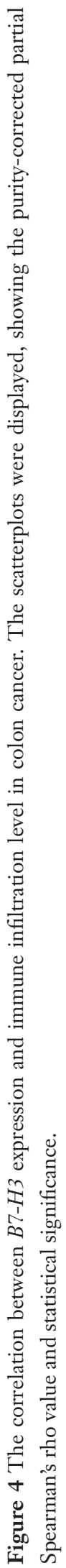

shorter survival time (55), which was consistent with our results. Effective therapeutic strategies against colon cancer are scarce. Therefore, it is imperative to seek a promising therapeutic target for anti-tumor drugs and also important to develop a prognostic tool to identify patients at high risk of mortality who require more attention and aggressive treatment. By leveraging publicly available transcriptional data for European colon cancers in TCGA and GEO cohorts and our in-door data for Asian colon cancers in the UNION cohort, we performed a three-phase study, which perhaps was the first trans-ethnic prognostic study of $\mathrm{B} 7-\mathrm{H3}$ expression on this population level. We first tested the prognostic effect of $\mathrm{B} 7-\mathrm{H}_{3}$ on survival in the European population, and then continuously validated its effect using another 2 independent European and Asian populations. Highly expressed $B 7-H 3$ was a significant and independent harmful factor to colon cancer survival, even after adjusting for effects of other immune checkpoint genes, correlating with these genes, and joint involvement in TIME. We also proposed an immune checkpoint prognostic risk score which significantly stratified patients into high and low risk groups, regardless of their characteristics.

The mechanism of $B 7-H 3$ expression affecting colon cancer survival still remains to be fully investigated. $\mathrm{B} 7-\mathrm{H} 3$ expression is often induced on immune cells $(56,57)$, which can mediate chemotherapeutic resistance and sensitivity to survival of patients $(58,59)$. It was also shown that $B 7$ $H 3$ promotes $V E G F A$ expression and angiogenesis, which dependents on the NF- $\kappa \mathrm{B}$ pathway in colorectal cancer (CRC), and CRC cell recruits regulatory $T$ cells to promote chemoresistance via NF- $\kappa \mathrm{B}$ signaling pathway (60). Besides, $B 7-H 3$ is also regulated by Th1, IL-4, IFN-gamma and TNF-alpha, which is an important cancer-promoting inflammatory molecule and significantly increases the release of soluble $\mathrm{B} 7-\mathrm{H} 3$ in colon cancer cell lines $(61,62)$. Meanwhile, the $B 7-H 3$ pathway has a dual role in contributing to the regulation of innate immune responses. On the one hand, $\mathrm{B} 7-\mathrm{H} 3$ co-stimulates innate immunity by augmenting proinflammatory cytokines release from lipopolysaccharide-stimulated monocytes or macrophages in both a Toll like receptor 4- and 2-dependent manner $(49,50,63)$. On the other hand, $B 7-H 3$ provides an additional mechanism allowing neuroblastoma cells, which protect them from natural killer cell-mediated lysis, to escape the control of immune response which may be associated with the expression of $\mathrm{B} 7-\mathrm{H} 3$ and multiple possibly related molecules, such as programmed cell death protein 1 (PD-1), cytotoxic T-lymphocyte-associated protein 


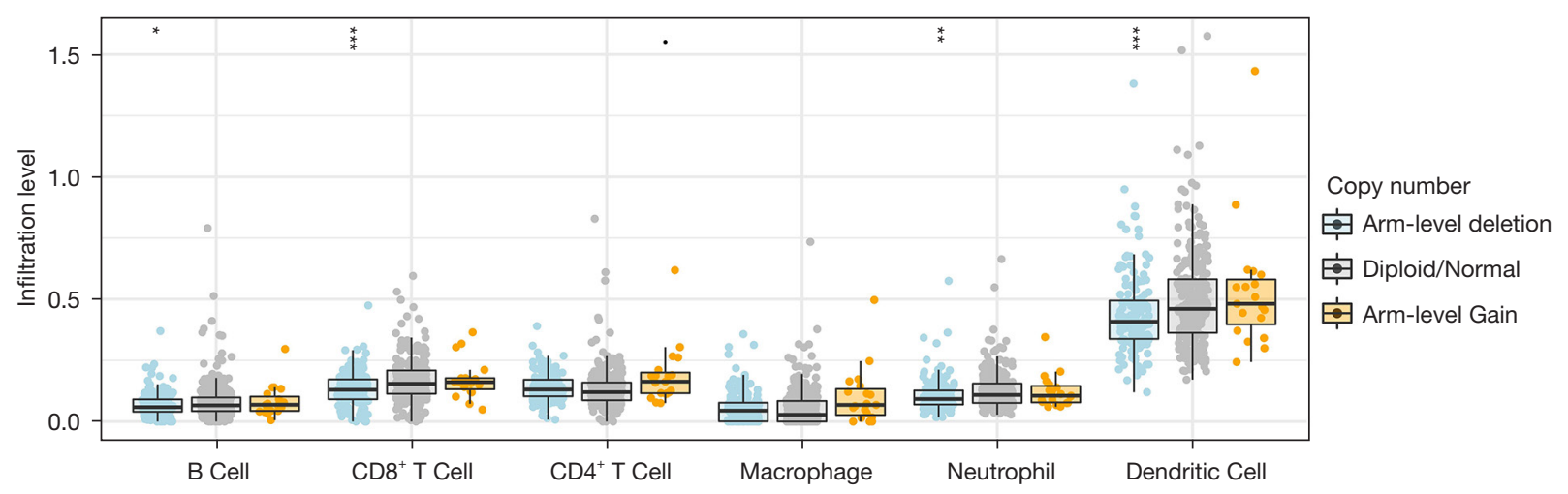

Figure 5 The comparison of tumor infiltration levels among tumors with different somatic copy number alterations of $B 7-H 3$. Copy number alternations were categorized into different groups, including deep deletion (-2), arm-level deletion (-1), diploid/normal (0), armlevel gain $(+1)$, and high amplification $(+2)$. Box plots are presented to show the distributions of each immune subset at each copy number status in COAD. The infiltration level for each category is compared with the normal using a two-sided Wilcoxon rank-sum test. ${ }^{\bullet}, \mathrm{P}<0.1$; *, $\mathrm{P}<0.05 ;$ **, $\mathrm{P}<0.01$; *** $\mathrm{P}<0.001$. COAD, colon adenocarcinoma.

4 (CTAL-4) or the presence of different types of cells associated with immune responses $(11,64)$. Nevertheless, the role of $\mathrm{B} 7-\mathrm{H3}$ in controlling the innate immunity is quite complex and requires more elucidation (65). Emerging evidence indicates that immune checkpoint blockade combined with radiotherapy acquires considerable success in multiple tumors $(66,67)$. On account of its important role in immune evasion and cancer progression, $B 7-H 3$ also possesses the capability to be a potential novel therapeutic target for colon cancer patients (68). Besides, $B 7-H 3$ also plays a non-immunological role in promoting tumor invasion and progression $(69,70)$. It was observed that the 3' UTR of B7-H3 transcript contains a target site for miR29 microRNA, and there is an inverse correlation between the expression of B7-H3 protein and miR29 levels, suggesting regulation of expression of this gene product by miR29. Experimental studies in mice showed that induction of colitis and water avoidance stress affect levels of Mir29a and Mir29b and intestinal permeability in wildtype mice (71). Despite inconsistent results and conflicting conclusions of existing prognostic studies of $\mathrm{B} 7-\mathrm{H3}$ (22,24-30,32-37), our study, with the largest sample size, confirmed that it was a significant and independent biomarker to colon cancer survival by several trans-ethnical validations.

Our study has several strengths. First, this was perhaps the largest prognostic study of $B 7-H 3$ in colon cancer patients on a population level. A 3-phase design was applied to control false positives and ensure the robustness of our results. The significant association observed in the discovery phase was further replicated in the validation phases I and II. Therefore, the probability of reporting the false positive result was strictly controlled under $0.0125 \%$ $(5 \% \times 5 \% \times 5 \%)$. Second, though population heterogeneity exists even among those of the same ethnicity, taking advantage of the RCS model, we successfully elucidated the nonlinear effect of $B 7-H 3$ expression on colon cancer survival in the TCGA European population and its approximate linear effect in GEO European population. Third, by trans-ethnic validation using an Asian population, we confirmed the robust association between $B 7-H_{3}$ expression and colon cancer survival again, meaning that this potential therapeutic target can be uniformly applied to all colon cancer patients worldwide. Fourth, by adjusting for other well-recognized immune checkpoint genes, B7$H 3$ was still a significant biomarker to colon cancer survival, indicating it was an independent prognostic factor. Finally, we proposed an immune checkpoint prognostic risk score of colon cancer using 8 genes, which can identify patients at high risk of mortality regardless of age, gender, and clinical stages, possible providing an effective tool aiding in clinical interventions.

We also acknowledge some limitations. First, the censoring rate is high in the TCGA and GEO cohorts, which resulted in a low statistical power in time-to-event data analysis. Regardless, we still observed significant results, indicating the association between $B 7-H 3$ expression and colon cancer survival was quite conservative. Second, several important clinical variables (e.g., status of complete tumor excision and tumor size) were not available in the TCGA and GEO cohorts, therefore a limited number of 
covariates were adjusted in the Cox proportional hazards regression model. We envision a better model adjusted for more clinical variables to achieve more precise estimates of $B 7-H 3$ effect and more accurate prognostic prediction ability in future studies. Finally, functional studies of $\mathrm{B} 7-\mathrm{H}_{3}$ are warranted to confirm its biological effect.

\section{Conclusions}

In summary, in the largest prognostic study of $B 7-H 3$ in colon cancer patients on population levels, we utilized a 3 -phase design to confirm that $B 7-H 3$ was a significant and independent biomarker of colon cancer prognosis. The proposed immune checkpoint prognostic risk score has the capability to identify colon cancers with high risk of mortality.

\section{Acknowledgements}

The authors express great thanks to patients and investigators who participated in TCGA and GEO projects for sharing the raw data.

Funding: This work was supported by the National Natural Science Foundation of China (81902378 to P Chi); Construction Project of Fujian Province Minimally Invasive Medical Center (2017-171 to P Chi); Startup Fund for Scientific Research, Fujian Medical University (2018XQ2025 to Y Gao); Joint Funds for the Innovation of Science and Technology of Fujian Province (2017Y9038, 2017Y9104) and Young and Middle-aged Backbone Training Project in the Health System of Fujian Province (2019-ZQN-45).

\section{Footnote}

Reporting Checklist: The authors have completed the REMARK reporting checklist. Available at https://dx.doi. org/10.21037/jgo-21-821

Data Sharing Statement: Available at https://dx.doi. org/10.21037/jgo-21-821

Conflicts of Interest: All authors have completed the ICMJE uniform disclosure form (available at https://dx.doi. org/10.21037/jgo-21-821). The authors have no conflicts of interest to declare.

Ethical Statement: The authors are accountable for all aspects of the work in ensuring that questions related to the accuracy or integrity of any part of the work are appropriately investigated and resolved. All procedures performed in this study involving human participants were in accordance with the Declaration of Helsinki (as revised in 2013). This study was approved by the institutional review board of Fujian Medical University Union Hospital (No. 2018YF024-01). All participants or their surrogates provided their written informed consent.

Open Access Statement: This is an Open Access article distributed in accordance with the Creative Commons Attribution-NonCommercial-NoDerivs 4.0 International License (CC BY-NC-ND 4.0), which permits the noncommercial replication and distribution of the article with the strict proviso that no changes or edits are made and the original work is properly cited (including links to both the formal publication through the relevant DOI and the license). See: https://creativecommons.org/licenses/by-nc-nd/4.0/.

\section{References}

1. Sung H, Ferlay J, Siegel RL, et al. Global Cancer Statistics 2020: GLOBOCAN Estimates of Incidence and Mortality Worldwide for 36 Cancers in 185 Countries. CA Cancer J Clin 2021;71:209-49.

2. Xie YH, Chen YX, Fang JY. Comprehensive review of targeted therapy for colorectal cancer. Signal Transduct Target Ther 2020;5:22.

3. Waldman AD, Fritz JM, Lenardo MJ. A guide to cancer immunotherapy: from $\mathrm{T}$ cell basic science to clinical practice. Nat Rev Immunol 2020;20:651-68.

4. Momtaz P, Postow MA. Immunologic checkpoints in cancer therapy: focus on the programmed death-1 (PD-1) receptor pathway. Pharmgenomics Pers Med 2014;7:357-65.

5. Pardoll DM. The blockade of immune checkpoints in cancer immunotherapy. Nat Rev Cancer 2012;12:252-64.

6. Marin-Acevedo JA, Dholaria B, Soyano AE, et al. Next generation of immune checkpoint therapy in cancer: new developments and challenges. J Hematol Oncol 2018;11:39.

7. Dougan M, Dranoff G, Dougan SK. Cancer immunotherapy: beyond checkpoint blockade. Annu Rev Cancer Biol 2019;3:55-75.

8. Rotte A. Combination of CTLA-4 and PD-1 blockers for treatment of cancer. J Exp Clin Cancer Res 2019;38:255.

9. He X, Xu C. Immune checkpoint signaling and cancer 
immunotherapy. Cell Res 2020;30:660-9.

10. Janakiram $M$, Shah UA, Liu $W$, et al. The third group of the B7-CD28 immune checkpoint family: HHLA2, TMIGD2, B7x, and B7-H3. Immunol Rev 2017;276:26-39.

11. Chapoval AI, Ni J, Lau JS, et al. B7-H3: a costimulatory molecule for $\mathrm{T}$ cell activation and IFN- $\gamma$ production. Nat Immunol 2001;2:269-74.

12. Liu F, Zhang T, Zou S, et al. B7-H3 promotes cell migration and invasion through the Jak2/Stat3/MMP9 signaling pathway in colorectal cancer. Mol Med Rep 2015;12:5455-60.

13. Du H, Hirabayashi K, Ahn S, et al. Antitumor Responses in the Absence of Toxicity in Solid Tumors by Targeting B7-H3 via Chimeric Antigen Receptor T Cells. Cancer Cell 2019;35:221-237.e8.

14. Yang M, Tang X, Zhang Z, et al. Tandem CAR-T cells targeting CD70 and B7-H3 exhibit potent preclinical activity against multiple solid tumors. Theranostics 2020;10:7622-34.

15. Souweidane MM, Kramer K, Pandit-Taskar N, et al. Convection-enhanced delivery for diffuse intrinsic pontine glioma: a single-centre, dose-escalation, phase 1 trial. Lancet Oncol 2018;19:1040-50.

16. Kang FB, Wang L, Jia HC, et al. B7-H3 promotes aggression and invasion of hepatocellular carcinoma by targeting epithelial-to-mesenchymal transition via JAK2/STAT3/Slug signaling pathway. Cancer Cell Int 2015;15:45.

17. Yang S, Wei W, Zhao Q. B7-H3, a checkpoint molecule, as a target for cancer immunotherapy. Int J Biol Sci 2020;16:1767-73.

18. Arigami T, Narita N, Mizuno R, et al. B7-h3 ligand expression by primary breast cancer and associated with regional nodal metastasis. Ann Surg 2010;252:1044-51.

19. Majzner RG, Theruvath JL, Nellan A, et al. CAR T Cells Targeting B7-H3, a Pan-Cancer Antigen, Demonstrate Potent Preclinical Activity Against Pediatric Solid Tumors and Brain Tumors. Clin Cancer Res 2019;25:2560-74.

20. Wang J, Chong KK, Nakamura Y, et al. B7-H3 associated with tumor progression and epigenetic regulatory activity in cutaneous melanoma. J Invest Dermatol 2013;133:2050-8.

21. Yamato I, Sho M, Nomi T, et al. Clinical importance of B7-H3 expression in human pancreatic cancer. Br J Cancer 2009; 101:1709-16.

22. Tang J, Jiang W, Liu D, et al. The comprehensive molecular landscape of the immunologic co-stimulator B7 and TNFR ligand receptor families in colorectal cancer: immunotherapeutic implications with microsatellite instability. Oncoimmunology 2018;7:e1488566.

23. Vogl UM, Öhler L, Rasic M, et al. Evaluation of Prognostic Immune Signatures in Patients with Breast, Colorectal and Pancreatic Cancer Receiving Chemotherapy. Anticancer Res 2017;37:1947-55.

24. Bin Z, Guangbo Z, Yan G, et al. Overexpression of B7-H3 in CD133+ colorectal cancer cells is associated with cancer progression and survival in human patients. J Surg Res 2014;188:396-403.

25. Hu X, Xu M, Hu Y, et al. B7-H3, Negatively Regulated by miR-128, Promotes Colorectal Cancer Cell Proliferation and Migration. Cell Biochem Biophys 2021;79:397-405.

26. Wu J, Wang F, Liu X, et al. Correlation of IDH1 and B7H3 expression with prognosis of CRC patients. Eur J Surg Oncol 2018;44:1254-60.

27. Mao Y, Chen L, Wang F, et al. Cancer cell-expressed B7-H3 regulates the differentiation of tumor-associated macrophages in human colorectal carcinoma. Oncol Lett 2017;14:6177-83.

28. Fan H, Zhu JH, Yao XQ. Prognostic significance of B7$\mathrm{H} 3$ expression in patients with colorectal cancer: A metaanalysis. Pak J Med Sci 2016;32:1568-73.

29. Zhang W, Acuna-Villaorduna A, Kuan K, et al. B7-H3 and PD-L1 Expression Are Prognostic Biomarkers in a Multiracial Cohort of Patients with Colorectal Cancer. Clin Colorectal Cancer 2021;20:161-9.

30. Zhang T, Jin Y, Jiang X, et al. Clinical and Prognostic Relevance of B7-H3 and Indicators of Glucose Metabolism in Colorectal Cancer. Front Oncol 2020;10:546110.

31. Ingebrigtsen VA, Boye $\mathrm{K}$, Tekle C, et al. B7-H3 expression in colorectal cancer: nuclear localization strongly predicts poor outcome in colon cancer. Int J Cancer 2012;131:2528-36.

32. Tang YC, Zhang Y, Zhou J, et al. Ginsenoside Rg3 targets cancer stem cells and tumor angiogenesis to inhibit colorectal cancer progression in vivo. Int $\mathrm{J}$ Oncol 2018;52:127-38.

33. Zhang C, Chen Y, Li F, et al. B7-H3 is spliced by SRSF3 in colorectal cancer. Cancer Immunol Immunother 2021;70:311-21.

34. Lu Z, Zhao ZX, Cheng P, et al. B7-H3 immune checkpoint expression is a poor prognostic factor in colorectal carcinoma. Mod Pathol 2020;33:2330-40.

35. Ingebrigtsen VA, Boye K, Nesland JM, et al. B7$\mathrm{H} 3$ expression in colorectal cancer: associations with clinicopathological parameters and patient outcome. BMC Cancer 2014;14:602. 
36. Mao Y, Sun J, Wang WP, et al. Clinical significance of costimulatory molecule B7-H3 expression on CD3(+) $\mathrm{T}$ cells in colorectal carcinoma. Chin Med J (Engl) 2013;126:3035-8.

37. Liu X, Wang F, Wu J, et al. Expression of CYP1B1 and B7-H3 significantly correlates with poor prognosis in colorectal cancer patients. Int J Clin Exp Pathol 2018;11:2654-64.

38. McShane LM, Altman DG, Sauerbrei W, et al. REporting recommendations for tumour MARKer prognostic studies (REMARK). Br J Cancer 2005;93:387-91.

39. Liao Q, Zeng Z, Guo X, et al. LPLUNC1 suppresses IL-6-induced nasopharyngeal carcinoma cell proliferation via inhibiting the Stat 3 activation. Oncogene 2014;33:2098-109.

40. Gauthier J, Wu QV, Gooley TA. Cubic splines to model relationships between continuous variables and outcomes: a guide for clinicians. Bone Marrow Transplant 2020;55:675-80.

41. Durrleman S, Simon R. Flexible regression models with cubic splines. Stat Med 1989;8:551-61.

42. Schoenfeld D. Partial residuals for the proportional hazards regression model. Biometrika 1982;69:239-41.

43. Rich JT, Neely JG, Paniello RC, et al. A practical guide to understanding Kaplan-Meier curves. Otolaryngol Head Neck Surg 2010;143:331-6.

44. Li T, Fu J, Zeng Z, et al. TIMER2.0 for analysis of tumor-infiltrating immune cells. Nucleic Acids Res 2020;48:W509-14.

45. Li T, Fan J, Wang B, et al. TIMER: A Web Server for Comprehensive Analysis of Tumor-Infiltrating Immune Cells. Cancer Res 2017;77:e108-10.

46. Qin S, Xu L, Yi M, et al. Novel immune checkpoint targets: moving beyond PD-1 and CTLA-4. Mol Cancer 2019;18:155.

47. Hajian-Tilaki K. Receiver Operating Characteristic (ROC) Curve Analysis for Medical Diagnostic Test Evaluation. Caspian J Intern Med 2013;4:627-35.

48. Hofmeyer KA, Ray A, Zang X. The contrasting role of B7-H3. Proc Natl Acad Sci U S A 2008;105:10277-8.

49. Zhang G, Wang J, Kelly J, et al. B7-H3 augments the inflammatory response and is associated with human sepsis. J Immunol 2010;185:3677-84.

50. Chen X, Quinn EM, Ni H, et al. B7-H3 participates in the development of experimental pneumococcal meningitis by augmentation of the inflammatory response via a TLR2dependent mechanism. J Immunol 2012;189:347-55.

51. Kontos F, Michelakos T, Kurokawa T, et al. B7-H3: An
Attractive Target for Antibody-based Immunotherapy. Clin Cancer Res 2021;27:1227-35.

52. Wang L, Zhao Y, Xu M, et al. Serum miR-1301-3p, miR-335-5p, miR-28-5p, and their target B7-H3 may serve as novel biomarkers for colorectal cancer. J BUON 2019;24:1120-7.

53. Yasui K, Kondou R, lizuka A, et al. Effect of preoperative chemoradiotherapy on the immunological status of rectal cancer patients. J Radiat Res 2020;61:766-75.

54. Wang J, Chen X, Xie C, et al. MicroRNA miR-29a Inhibits Colon Cancer Progression by Downregulating B7-H3 Expression: Potential Molecular Targets for Colon Cancer Therapy. Mol Biotechnol 2021;63:849-61.

55. Shi T, Ma Y, Cao L, et al. B7-H3 promotes aerobic glycolysis and chemoresistance in colorectal cancer cells by regulating HK2. Cell Death Dis 2019;10:308.

56. Picarda E, Ohaegbulam KC, Zang X. Molecular Pathways: Targeting B7-H3 (CD276) for Human Cancer Immunotherapy. Clin Cancer Res 2016;22:3425-31.

57. Wang Y, Zhang B, Huang H, et al. An improved method to build lung cancer PDX models by surgical resection samples and its association with B7-H3 expression. Transl Cancer Res 2019;8:2848-57.

58. Yin Y, Yao S, Hu Y, et al. The Immune-microenvironment Confers Chemoresistance of Colorectal Cancer through Macrophage-Derived IL6. Clin Cancer Res 2017;23:7375-87.

59. Ye L, Zhang T, Kang Z, et al. Tumor-Infiltrating Immune Cells Act as a Marker for Prognosis in Colorectal Cancer. Front Immunol 2019;10:2368.

60. Wang R, Ma Y, Zhan S, et al. B7-H3 promotes colorectal cancer angiogenesis through activating the NF-kappaB pathway to induce VEGFA expression. Cell Death Dis 2020;11:55.

61. Sun J, Chen LJ, Zhang GB, et al. Clinical significance and regulation of the costimulatory molecule $\mathrm{B} 7-\mathrm{H} 3$ in human colorectal carcinoma. Cancer Immunol Immunother 2010;59:1163-71.

62. Meng F, Yang M, Chen Y, et al. miR-34a induces immunosuppression in colorectal carcinoma through modulating a SIRT1/NF-kappaB/B7-H3/TNF-alpha axis. Cancer Immunol Immunother 2021;70:2247-59.

63. Li Y, Huang J, Foley NM, et al. B7H3 ameliorates LPSinduced acute lung injury via attenuation of neutrophil migration and infiltration. Sci Rep 2016;6:31284.

64. Castriconi R, Dondero A, Augugliaro R, et al. Identification of 4Ig-B7-H3 as a neuroblastomaassociated molecule that exerts a protective role from 
an NK cell-mediated lysis. Proc Natl Acad Sci U S A 2004;101:12640-5.

65. Yi KH, Chen L. Fine tuning the immune response through B7-H3 and B7-H4. Immunol Rev 2009;229:145-51.

66. Patel SA, Minn AJ. Combination Cancer Therapy with Immune Checkpoint Blockade: Mechanisms and Strategies. Immunity 2018;48:417-33.

67. Qi Y, Liu B, Sun Q, et al. Immune Checkpoint Targeted Therapy in Glioma: Status and Hopes. Front Immunol 2020;11:578877.

68. Ye Q, Liu J, Xie K. B7 Family Proteins in Cancer Progression: Immunological and Non-Immunological Functions. J Cancer Treatment Diagn 2019;3:1-6.

Cite this article as: Gao Y, Xu Y, Gao M, Huang A, Chi P. A three-phase trans-ethnic study reveals $B 7-H 3$ expression is a significant and independent biomarker associated with colon cancer overall survival. J Gastrointest Oncol 2021;12(6):2891-2905. doi: 10.21037/jgo-21-821
69. Zhou WT, Jin WL. B7-H3/CD276: An Emerging Cancer Immunotherapy. Front Immunol 2021;12:701006.

70. McCartney DL, Walker RM, Morris SW, et al. Identification of polymorphic and off-target probe binding sites on the Illumina Infinium MethylationEPIC BeadChip. Genom Data 2016;9:22-4.

71. Zhou Q, Costinean S, Croce CM, et al. MicroRNA 29 targets nuclear factor-kappaB-repressing factor and Claudin 1 to increase intestinal permeability. Gastroenterology 2015;148:158-69.e8.

(English Language Editor: J. Jones) 

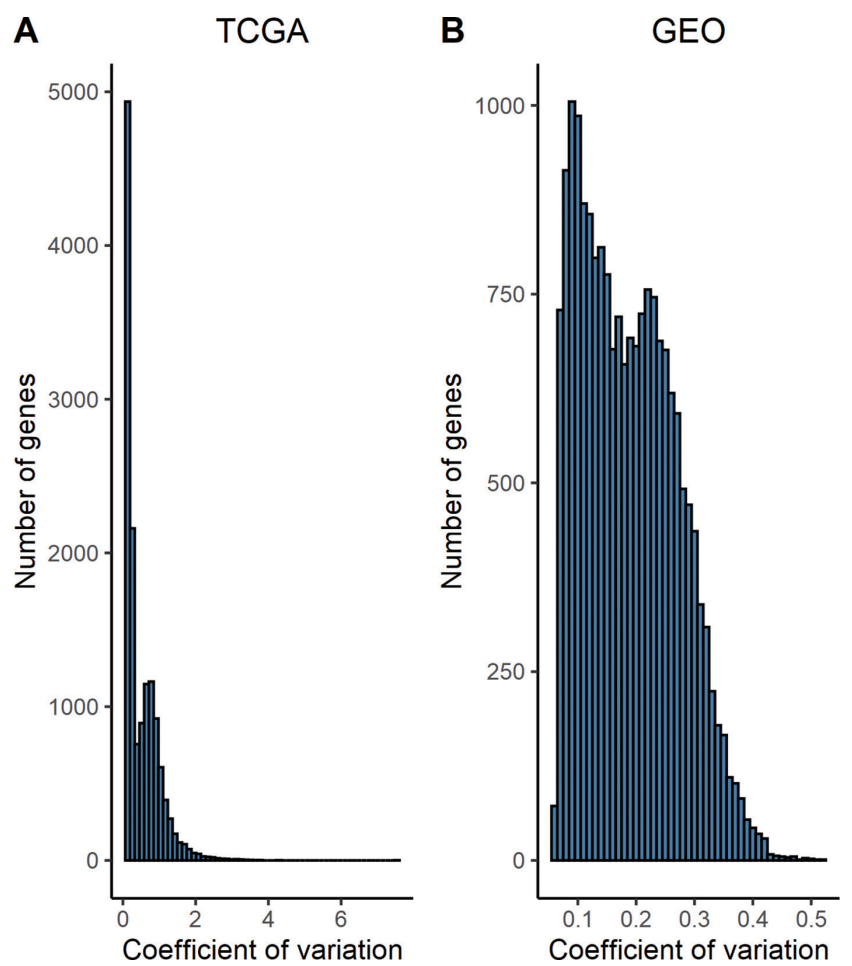

Figure $\mathbf{S 1}$ The distribution of coefficient of variation values of $B 7-H 3$ expression in TCGA and GEO cohorts. 
Table S1 Association results of each variable derived from univariate Cox proportional hazards model in TCGA, GEO and UNION cohorts

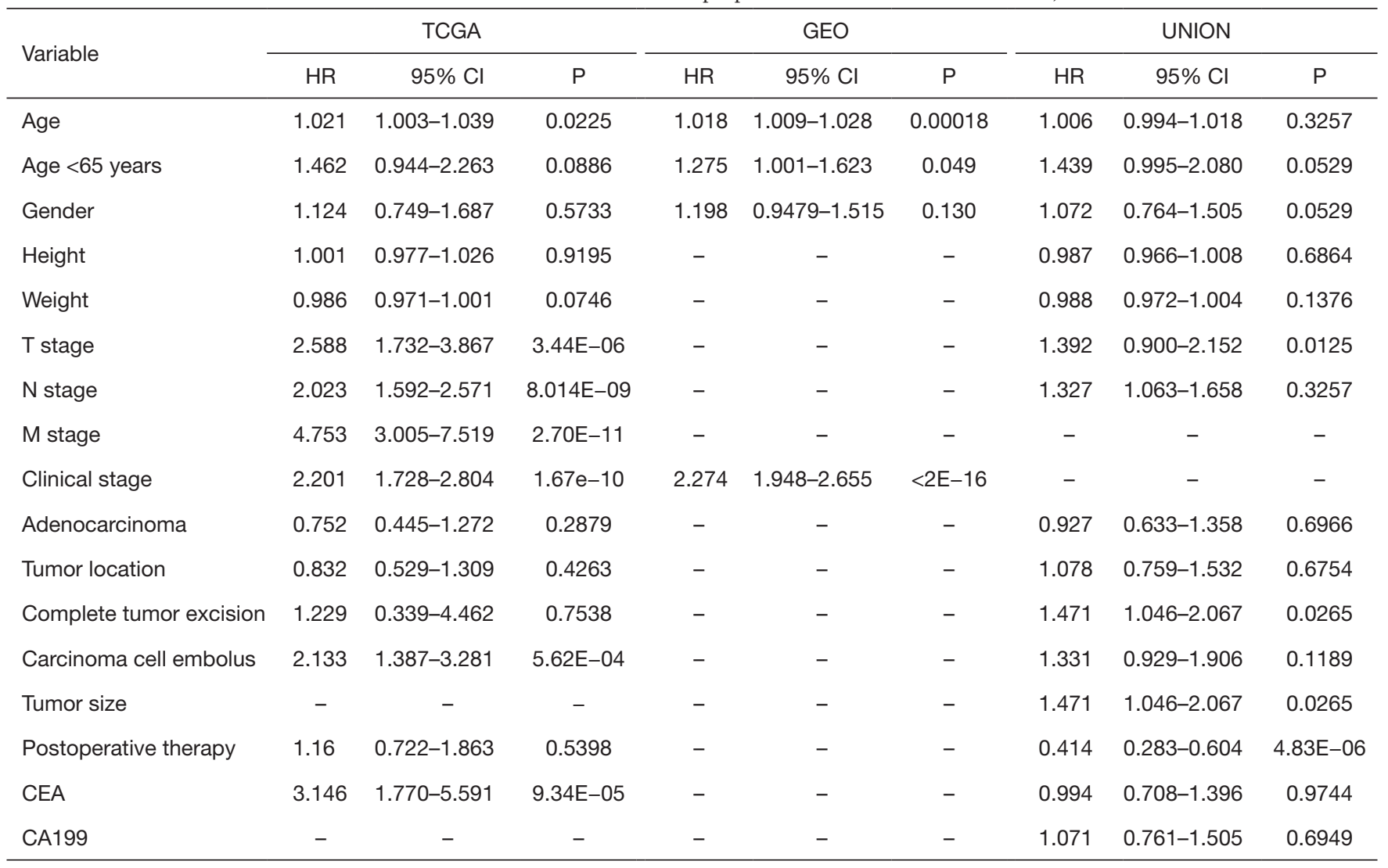

T, tumor; N, node; M, metastasis; CEA, carcinoembryonic antigen; CA199, carbohydrate antigen 199; HR, hazard ratio; Cl, confidence interval; TCGA, The Cancer Genome Atlas; GEO, Gene Expression Omnibus; UNION, Fujian Medical University Union Hospital.

Table S2 Association results of covariates derived from multivariate Cox proportional hazards model in TCGA, GEO and UNION cohorts

\begin{tabular}{|c|c|c|c|c|c|c|c|c|c|}
\hline Variable & \multicolumn{3}{|c|}{ TCGA } & \multicolumn{3}{|c|}{ GEO } & \multicolumn{3}{|c|}{ UNION } \\
\hline Age & 1.033 & $1.014-1.052$ & $6.38 \mathrm{E}-04$ & 1.027 & $1.016-1.039$ & $1.37 \mathrm{E}-06$ & 1.004 & $0.992-1.016$ & 0.4969 \\
\hline Gender & 0.964 & $0.631-1.473$ & 0.8663 & 1.378 & $1.080-1.757$ & 0.0098 & 0.987 & $0.699-1.393$ & 0.9412 \\
\hline T stage & - & - & - & - & - & - & 1.214 & $0.772-1.907$ & 0.4012 \\
\hline Clinical stage & 2.386 & $1.866-3.052$ & 4.34E-12 & 2.455 & $2.068-2.916$ & $<2 \mathrm{E}-16$ & - & - & - \\
\hline
\end{tabular}

Clinical stage which composed of T stage, $\mathrm{N}$ stage and $\mathrm{M}$ stage was included in the model in GEO and TCGA cohort, respectively. T stage and M stage were included in UNION cohort, since all subjects were in M1 stage. Significant variables were screened out by step forward regression model with $\mathrm{P}$ value of entry $\leq 0.05$ and $\mathrm{P}$ value of remove $>0.05$ in TCGA, GEO and UNION, respectively. Covariates were defined as significant variables in any one of cohorts, along with two demographic variables (age and gender) common adjusted in COAD prognostic study. T, tumor; N, node; HR, hazard ratio; Cl, confidence interval; TCGA, The Cancer Genome Atlas; GEO, Gene Expression Omnibus; UNION, Fujian Medical University Union Hospital. 

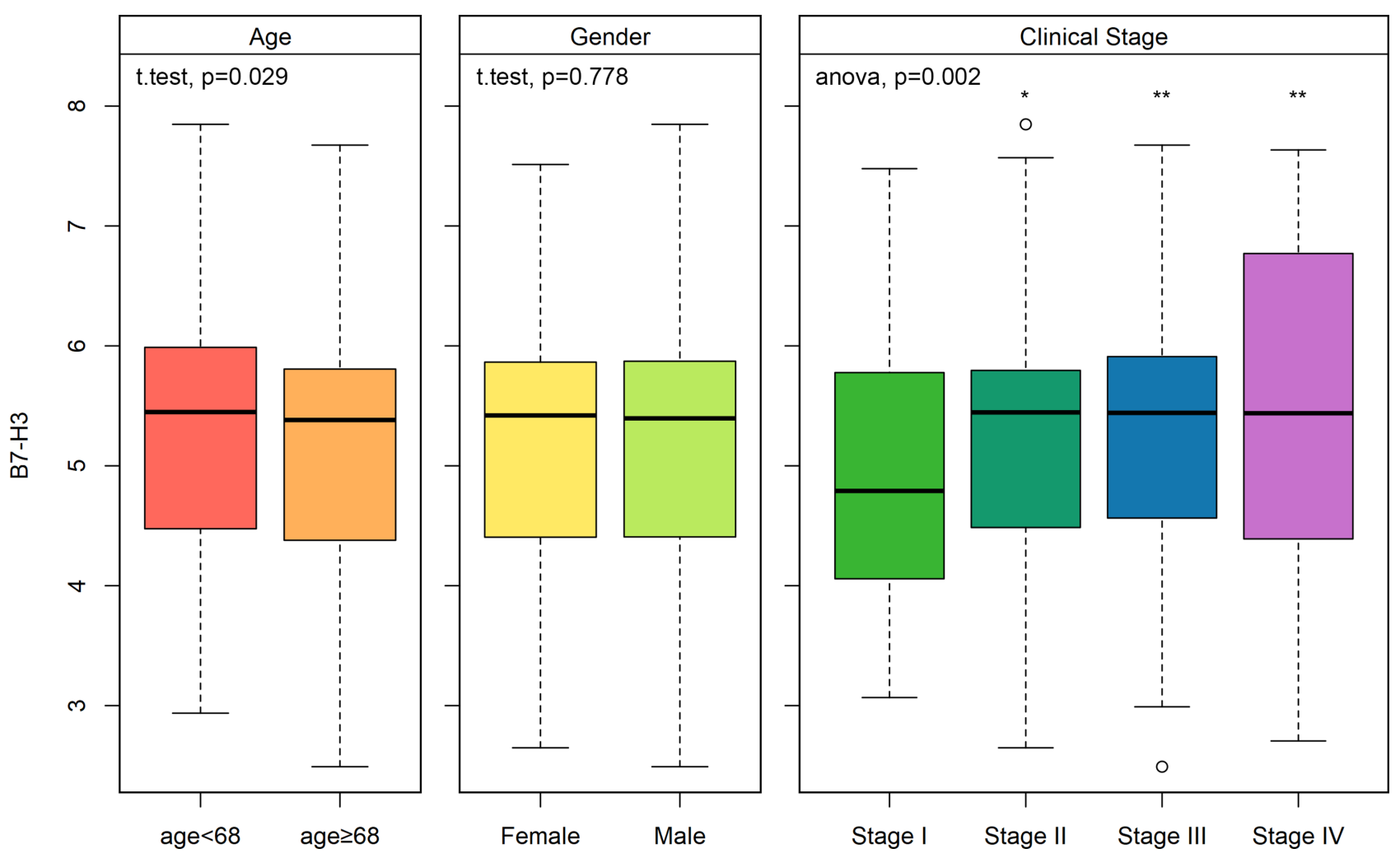

Figure S2 Boxplot of B7-H3 versus clinicopathological factors using all subjects from TCGA and GEO cohorts. The clinical stage for each category was compared with the stage I using a two-sided t test. *, $\mathrm{P}<0.05$; **, $\mathrm{P}<0.01$. 

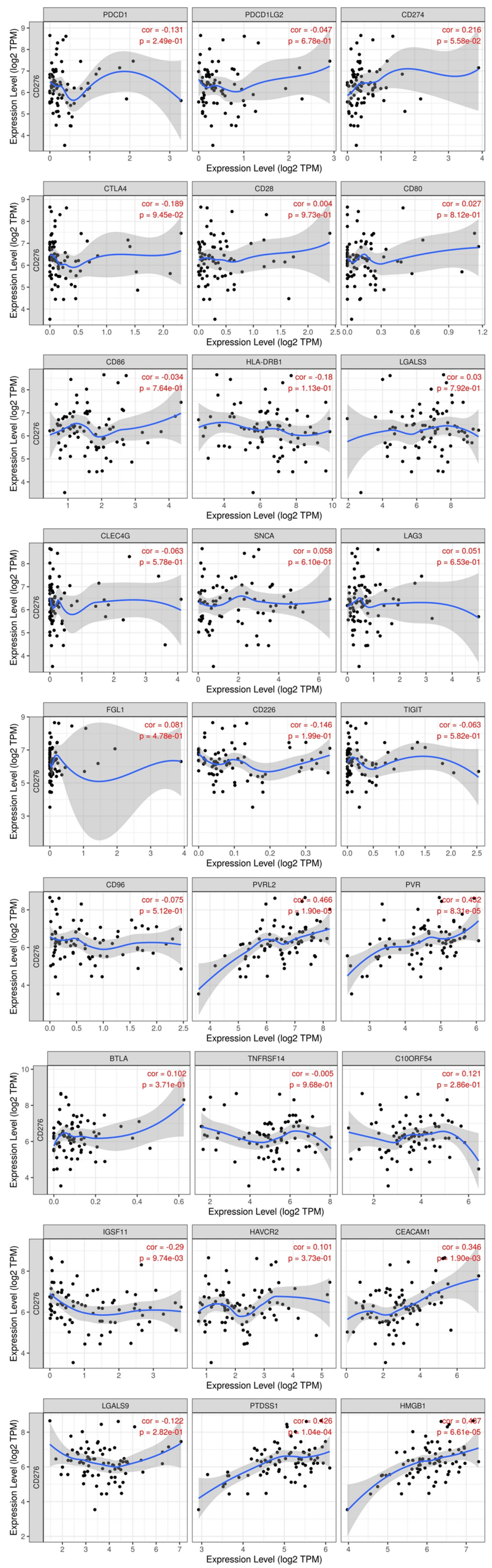

Figure 33 The correlation of between $B 7-H 3$ expression and immune checkpoint genes in colon cancer patients. 
Table S3 The multivariate Cox proportional hazards model using demographic, clinical characteristics and immune checkpoint genes

\begin{tabular}{lccc}
\hline \multirow{2}{*}{ Variable } & \multicolumn{2}{c}{ TCGA and GEO } & P \\
\cline { 2 - 3 } Age & HR & $1.018-1.038$ & $3.03 \mathrm{E}-08$ \\
Gender & 1.028 & $1.031-1.642$ & 0.0264 \\
Clinical stage & 1.302 & $1.880-2.543$ & $3.05 \mathrm{E}-24$ \\
Study site & 2.187 & $0.685-1.115$ & 0.2779 \\
B7-H3 & 0.874 & $1.067-2.022$ & 0.0184 \\
CTLA4 & 1.469 & $0.255-0.681$ & $4.79 \mathrm{E}-04$ \\
LAG3 & 0.416 & $1.128-1.900$ & 0.0042 \\
CD80 & 1.464 & $1.080-2.875$ & 0.0233 \\
LGALS9 & 1.762 & $0.611-0.861$ & $2.53 \mathrm{E}-04$ \\
HMGB1 & 0.725 & $0.64-0.988$ & 0.0383 \\
HLA-DOA & 0.795 & $1.110-2.061$ & 0.0088 \\
HLA-DPB1 & 1.512 & $0.612-0.964$ & 0.0226 \\
\hline All imm & 0.768 & &
\end{tabular}

All immune checkpoint genes were screened out by back forward stepwise regression model adjusted for age, gender, clinical stage, study site and B7-H3, with $\mathrm{P}$ value of entry $\leq 0.05$ and $\mathrm{P}$ value of remove $>0.05$. HR, hazard ratio; Cl, confidence interval; TCGA, The Cancer Genome Atlas; GEO, Gene Expression Omnibus; UNION, Fujian Medical University Union Hospital.
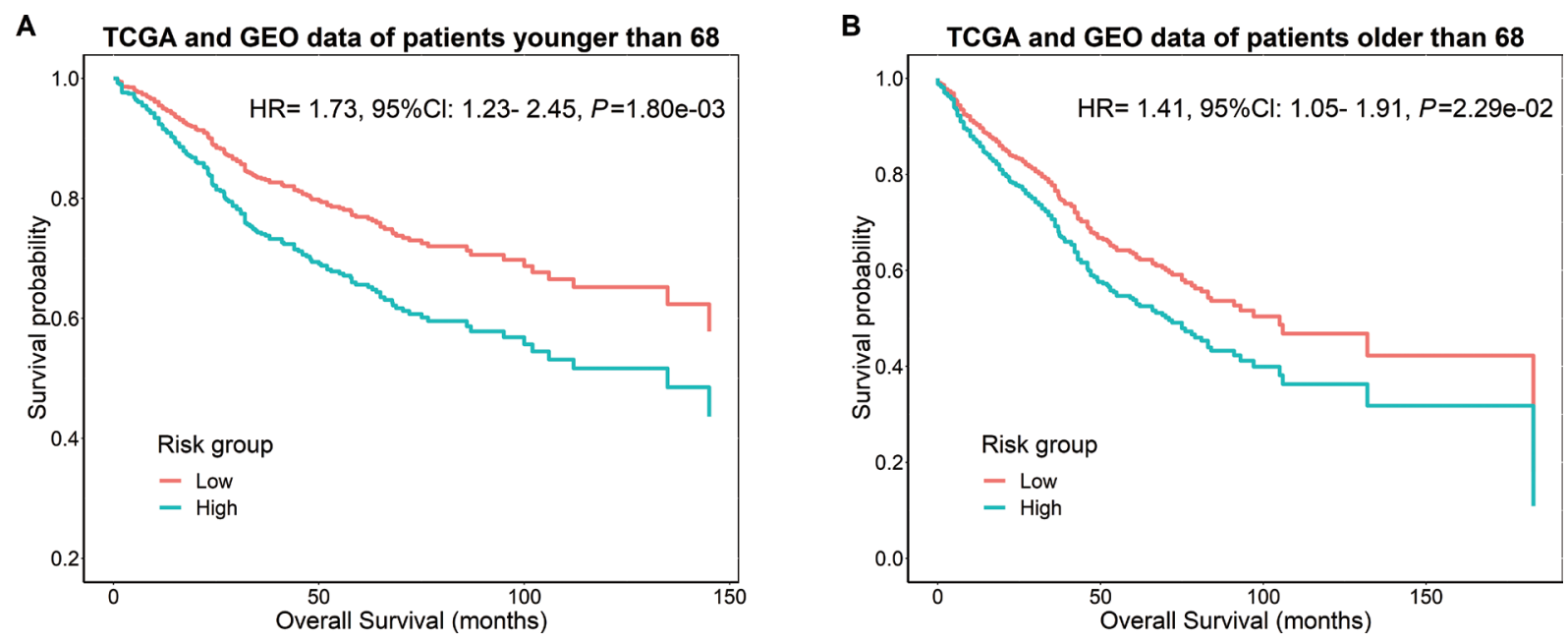

Figure S4 Kaplan-Meier survival curves for patients with high and low level of immune checkpoint prognostic risk score stratified by age using all subjects from TCGA and GEO cohorts. 

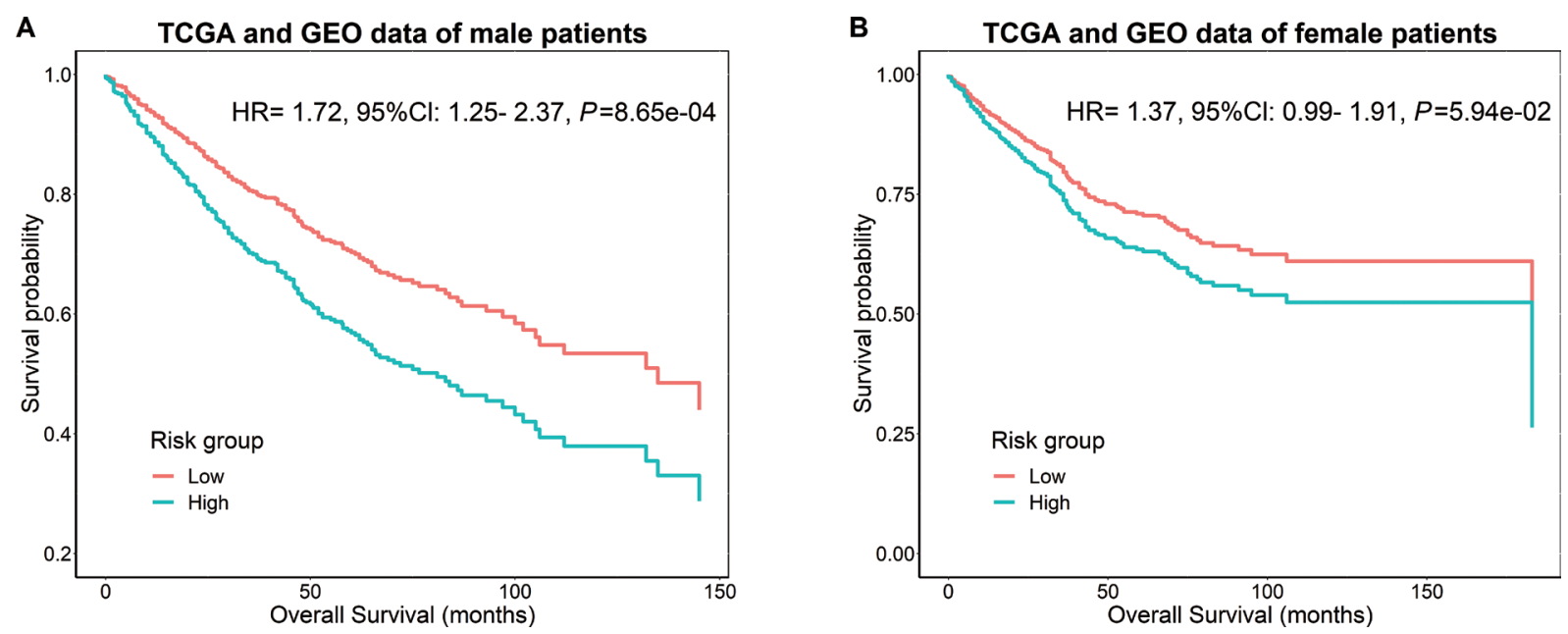

Figure S5 Kaplan-Meier survival curves for patients with high and low level of immune checkpoint prognostic risk score stratified by gender using all subjects from TCGA and GEO cohorts.
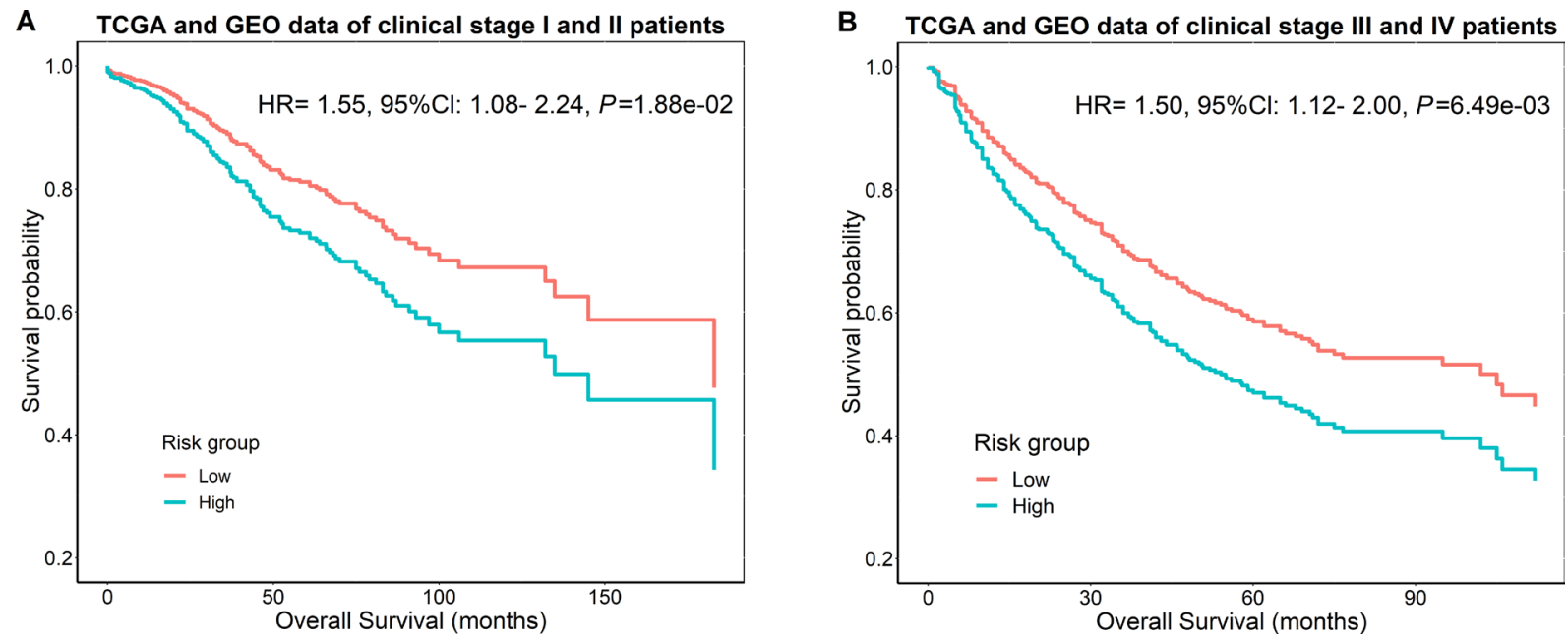

Figure S6 Kaplan-Meier survival curves for patients with high and low level of immune checkpoint prognostic risk score stratified by clinical stage using all subjects from TCGA and GEO cohorts. 
(A) ROC curve for prediction model of 3-year survival

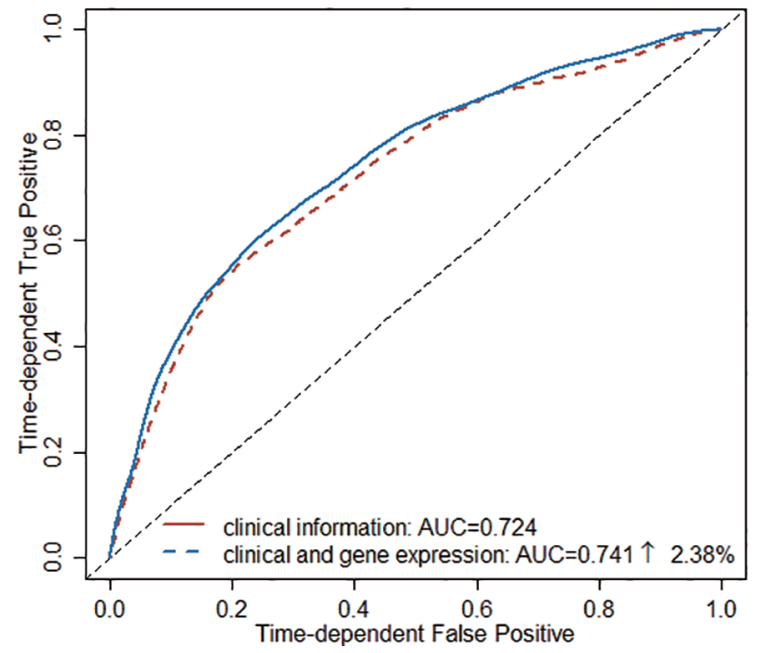

(B) ROC curve for prediction model of 5-year survival

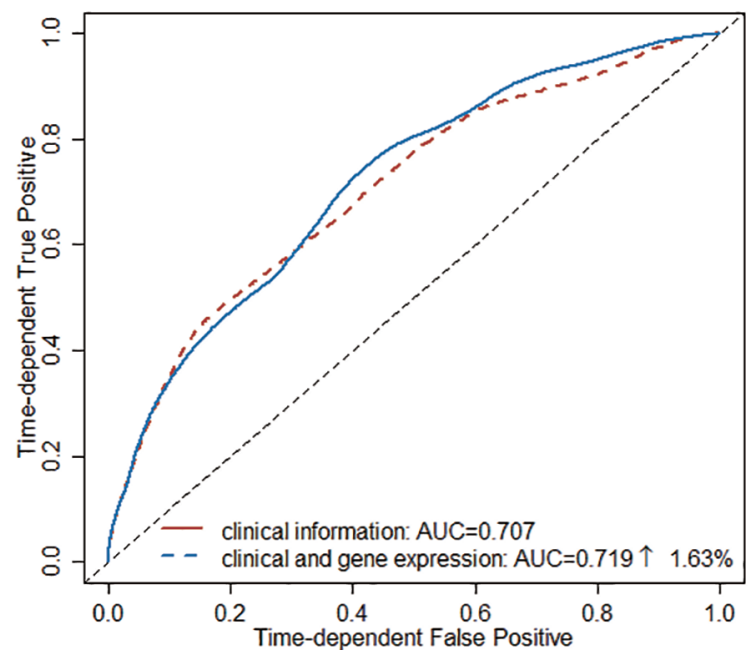

Figure S7 The time-dependent ROC of prognostic prediction model of 3- and 5-year overall survival, respectively. 